\title{
Histologic assessment of the biological effects after speedy surgical orthodontics in a beagle animal model: a preliminary study
}

\author{
Hong-Suk Kim, DDS, MSD, PhD, ${ }^{\text {a }}$ Young-Jun Lee, DDS, MSD, PhD, ${ }^{\mathrm{b}}$ Young-Guk Park, DDS, MSD, PhD, ${ }^{\mathrm{c}}$ \\ Kyu-Rhim Chung, DDS, MSD, PhD, ${ }^{\mathrm{d}}$ Yoon-Goo Kang, DDS, MSD, PhD, \\ HyeRan Choo, DDS, MSD, ${ }^{\mathrm{f}}$ Seong-Hun Kim, DDS, MSD, $\mathrm{PhD}^{\mathrm{g}}$
}

\begin{abstract}
Objective: Speedy surgical orthodontics (SSO), an innovative orthodontic treatment, involves the application of orthopedic forces against temporary skeletal anchorage devices following perisegmental corticotomy to induce movement of specific dental segments. Herein, we report the biological effects of SSO on the teeth and periodontal structures. Methods: Five beagle dogs were divided into 2 groups and their 6 maxillary incisors were retracted en masse by applying $500 \mathrm{~g}$ orthopedic force against a single palatal mini-plate. Retraction was performed without and with perisegmental corticotomy in groups I and II, respectively. All animals were killed on the 70th day, and their periodontal structures were processed for histologic analyses and scanning electronic microscopy (SEM). The linear distance between the third maxillary incisor and canine was used as a benchmark to quantify the retraction amount. Results: Retraction was markedly faster and retraction amount greater in group II than in Group I. Surprisingly, Group II did not show any root resorption despite extensive retraction, while Group I showed prominent root surface irregularities. Similarly, SEM showed multiple resorption lacunae in Group I, but not in Group II. Conclusions: SSO is an effective and favorable orthodontic approach for major en masse retraction of the maxillary anterior teeth. (Korean J Orthod 2011;41(5):361-370)
\end{abstract}

Key words: Speedy surgical orthodontics, Perisegmental corticotomy, Compression osteogenesis, en masse retraction, Root resorption, Mini-plate, Temporary skeletal anchorage device

${ }^{a}$ Postgraduate Student, ${ }^{b}$ Clinical Associate Professor, ${ }^{\circ}$ Professor and Chairman, ${ }^{\circ}$ Assistant Professor, ${ }^{9}$ Associate Professor, Department of Orthodontics, School of Dentistry, Kyung Hee University dProfessor and Chairman, Department of Dentistry, School of Medicine. Ajou University.

'Director, Department of Craniofacial Orthodontics, Childrens' Hospital of Phildelphia.

Corresponding author: Seong-Hun Kim.

Department of Orthodontics, School of Dentistry, Kyung Hee University, 1 Hoeigi-dong, Dongdaemun-gu, Seoul 130-701, Korea.

+82-2-958-9390: e-mail, bravortho@hanmail.net.

Received November 24, 2010; Last Revision June 7, 2011; Accepted June 7, 2011.

http://dx. doi.org/10.4041/kjod.2011.41.5.361

\section{INTRODUCTION}

Orthodontic treatment in adults has various limitations, including a slower rate of tooth movement, increased risk of root resorption, and higher relapse tendency due to thicker cortical bone and less vascularization. To overcome these limitations, various surgical techniques have been developed to facilitate rapid dentofacial movement for esthetic improvement. ${ }^{1-3}$ In addition to orthognathic surgery to improve facial esthetics, various other surgical techniques have been introduced as conjunctive measures for orthodontic treatment.

One of the earliest surgical techniques, introduced 
by Bryan in 1892, was designed to correct dental malocclusion and promote rapid tooth movement. This technique involved an osteotomy that removed the cortical bone believed to be a major interfering factor for tooth movement. ${ }^{4}$ Cunningham subsequently published a case report wherein a similar method of corticotomy was used to align teeth. ${ }^{5}$ Osteotomy is known to be associated with excessive bleeding. Hence, in 1959, Kole reported that corticotomy was a preferred method since it maintained the integrity of trabecular bone and promoted rapid and effective tooth movement, while causing less complications to the dentition or periodontium. ${ }^{6} \mathrm{He}$ focused on the advantage of corticotomy, in which the trabecular bone remains intact, to achieve rapid tooth movement without compromising tooth vitality. In parallel, Wilcko et al. ${ }^{4,8}$ recently introduced a new technique, namely, accelerated osteogenic orthodontics to facilitate orthodontic tooth movement. He suggested that the bone turnover rate is accelerated by corticotomy that allows rapid physiologic tooth movement following the application of orthodontic force.

On the other hand, Suya suggested a different perspective for the increased orthodontic efficiency with corticotomy. He suggested that the teeth embedded in the corticotomized bony segment function as a handle and control the movement of the segmented dental block, thereby facilitating orthodontic tooth movement. ${ }^{9}$ On the basis of Suya's concept, Chung et al. introduced a novel treatment concept, namely, speedy surgi- cal orthodontics (SSO), in 1999. Their method involved a perisegmental corticotomy around the maxillary posterior dental segment, followed by an orthopedic intrusion against an orthodontic palatal mini-plate. ${ }^{10}$ This treatment approach can be used to orthodontically correct an anterior openbite, without requiring orthognathic surgery. ${ }^{11,12}$

Although many previous clinical studies have described the effectiveness of the SSO protocol, its biological effects have been less understood since it is considered a seemingly aggressive approach to increase the orthodontic treatment efficiency. The current preliminary in vivo study therefore aimed to characterize and elucidate the effects of the SSO method on the dental root surface and periodontal structures after en masse retraction of the maxillary anterior segment.

\section{MATERIAL AND METHODS}

This animal study was approved by the committee on Animal Research at the Kyung Hee University, Dental Hospital. Five male beagle dogs (mean age, 18 months; body weight, 10 - $11 \mathrm{~kg}$ ) were used. The dogs had a good overall health and were carefully screened to exclude dental caries or periodontal diseases. The animals were fed a liquid diet throughout the experiment.

The animals were divided into 2 groups. Animals A and $\mathrm{B}$ were assigned to Group I (control group) and animals $\mathrm{C}, \mathrm{D}$, and $\mathrm{E}$ were assigned to Group II (expe-
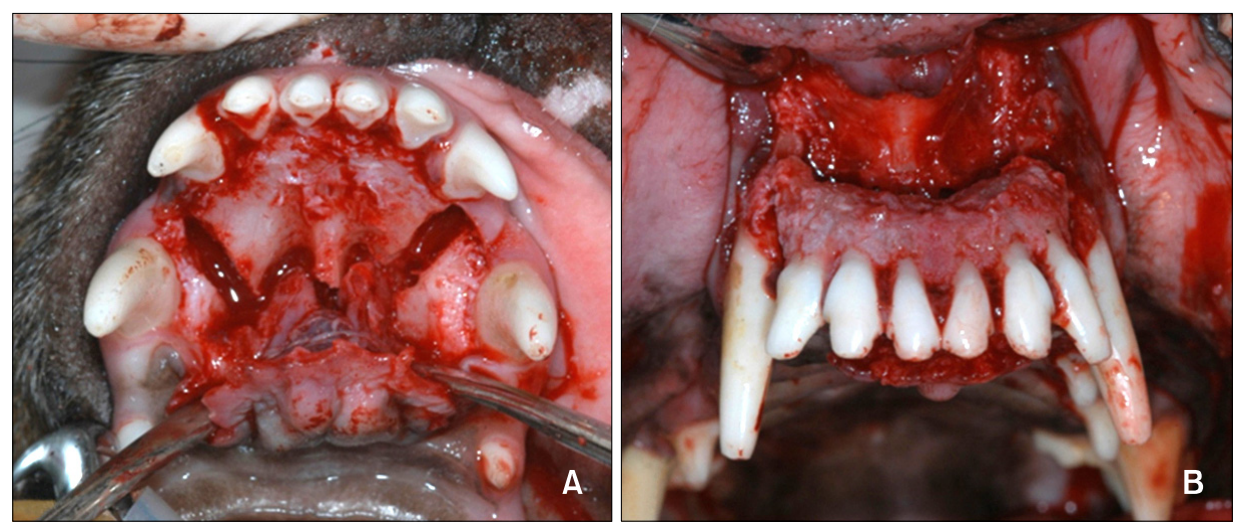

Fig 1. Surgical cuts for perisegmental corticotomy between the maxillary 3rd incisor and canine. A, Palatal corticotomy; B, labial corticotomy. 
rimental group). Perisegmental corticotomy was performed in the animals of Group II under general anesthesia (1 : 1 mixture of $0.22-0.44 \mathrm{~mL} / \mathrm{kg}$ ketamine and $1.5 \mathrm{~mL} / 10 \mathrm{~kg}$ xylazine) by using a local anesthetic (2\% lidocaine with $1: 100,000$ epinephrine). The perisegmental corticotomy was completed in a 2-step procedure such that the palatal corticotomy was performed 7 days before buccal corticotomy (Fig 1). The entire corticotomy line comprised 2 vertical incisions between both the maxillary canine and third incisor and a horizontal cut 2 - $3 \mathrm{~mm}$ gingival to the longest root connecting the vertical cuts on each side. Unlike anterior segmental osteotomy in which 1 segment is completely separated from the other parts of bone, corticotomy was primarily used to detach only the bony segment at the cortical bone level. The mucosae were completely elevated to establish maximum visualization of the corticotomy surgical site. Two corticotomies (1 buccal and 1 lingual) were performed at 2 time points with a 1-week interval to optimize the blood supply. A lowspeed engine with a 4-mm diameter round bur was used to ensure cortical bone separation while minimizing the perforation into the medullary bone. The cutting depth into the medullary bone was visually monitored by noting the bleeding point during corticotomy. In some cases, bone marrow may have been cut unintentionally; care was taken to keep the majority of the bone marrow intact. A palatal mini-plate (KLS Martin Co., Tuttlingen, Germany) was placed on the midpalatal suture area of all the animals on the tenth day of the experiment. Gentamycin $(0.08-0.1 \mathrm{~mL} / \mathrm{kg})$ was injected for 3 days to prevent infection following corti-

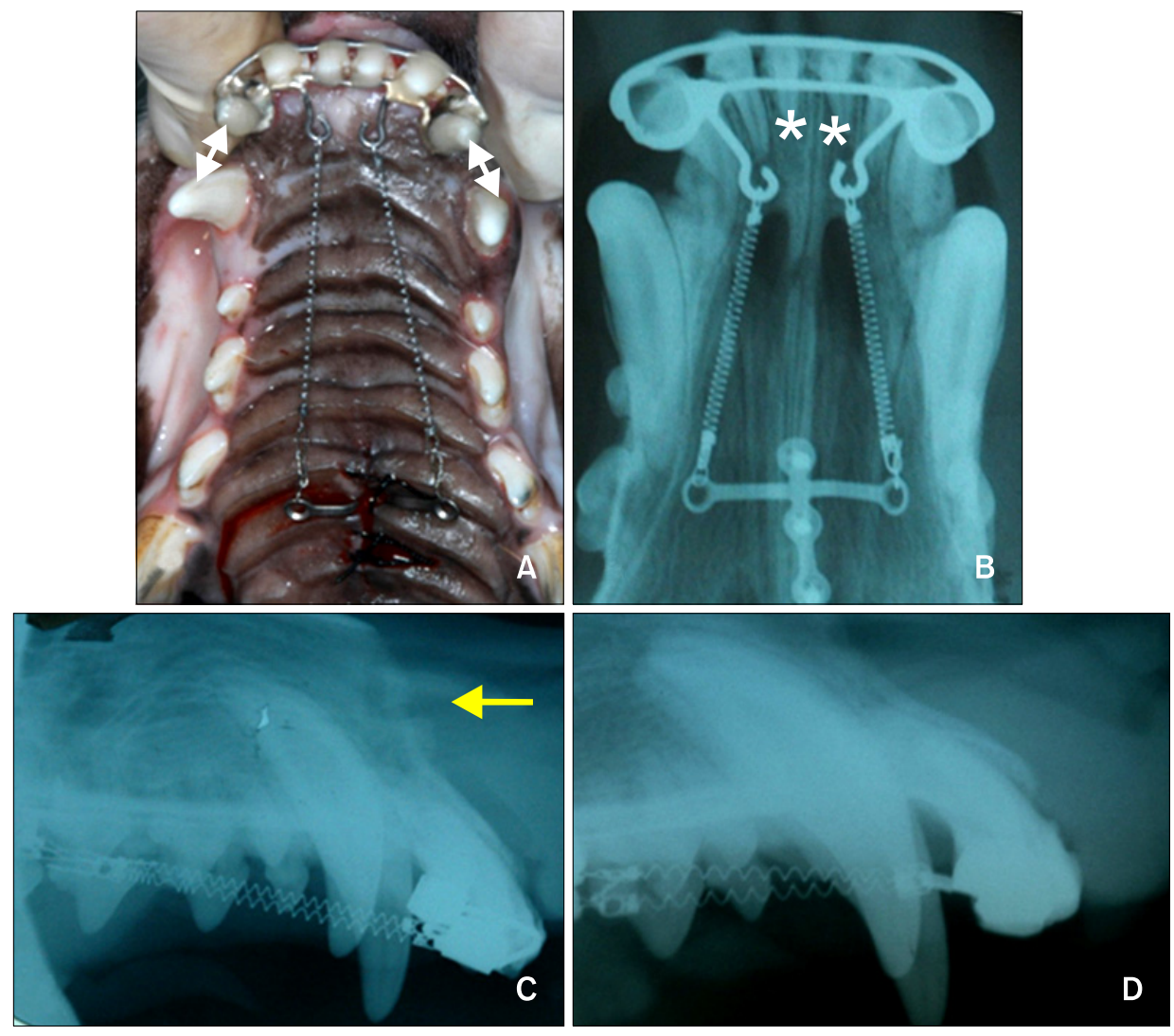

Fig 2. A, Intraoral photograph and; B, occlusal radiograph of the orthodontic appliances used for tooth movement immediately after force application using the modified C-lingual retractor and C-plate combined appliance; C, lateral cephalogram at the time of initial force application; D, lateral cephalogram after retraction. Distance measured (white arrow) and teeth used for histologic evaluation $\left(^{*}\right)$ are shown, and the arrow indicates the corticotomy site. 
cotomy and palatal mini-plate placement. A subsequent pulpotomy and occlusal reduction of the mandibular bicuspids was performed to avoid any potential occlusal interference that may occur during en masse retraction of the maxillary incisors. Each beagle was secured by a neck collar to restrict movement and avoid any damage to the intra-oral appliances.

On the 14th day of the experiment, a palatal retractor was placed on the palatal surface of the 6 maxillary incisors in both the groups (Fig 2A and B). ${ }^{13,14}$ Two NiTi closed-coil springs (250 g on each side) were used to produce a total of $500 \mathrm{~g}$ of constant continuous retraction force. Intraoral photographs and occlusal and lateral cephalometric radiographs were obtained at the beginning as well as on the completion of active retraction (Fig 2B to D). The amount of retraction was calculated by subtracting the post-retraction distance between the third incisor and canine from the pre-retraction distance. Distance between the third incisor and canine was intraorally measured using a digital caliper (Absolute Digimatic, Mitutoyo, Kawa- saki, Japan).

Animal E in Group II developed a maxillary fracture on the fourth day of active retraction and was excluded from the experiment. The remaining animals were euthanized by injecting xylazine (Rompun, Bayer, Monheim, Germany) and ketamine (Yuhan Co., Seoul, Korea) on the 70th day of the experiment (Fig 3). Next, the maxillae were dissected, split in half, and immediately fixed in $10 \%$ formalin. The teeth of the right maxilla were immersed in $2.5 \%$ glutaraldehyde, and the left maxillary teeth and periodontal tissues were decalcified using $5 \%$ nitric acid for 4 weeks. The specimens were then vacuum-embedded in paraffin and sectioned (thickness, $4 \mu \mathrm{m}$ ) along the longitudinal axis in a labiolingual direction. The specimens were stained with hematoxylin and eosin and photographed at $\times 10, \times 40, \times 100$, and $\times 400$ magnifications using Kappa ImageBase v.4.5.2 (Kappa opto-electronics $\mathrm{GmbH}$, Gleichen, Germany). The right maxillary teeth were stirred in a mixture of $0.1 \%$ collagenase and $1 \%$ phosphate-buffered saline (PBS) for $7 \mathrm{~h}$ and then dried

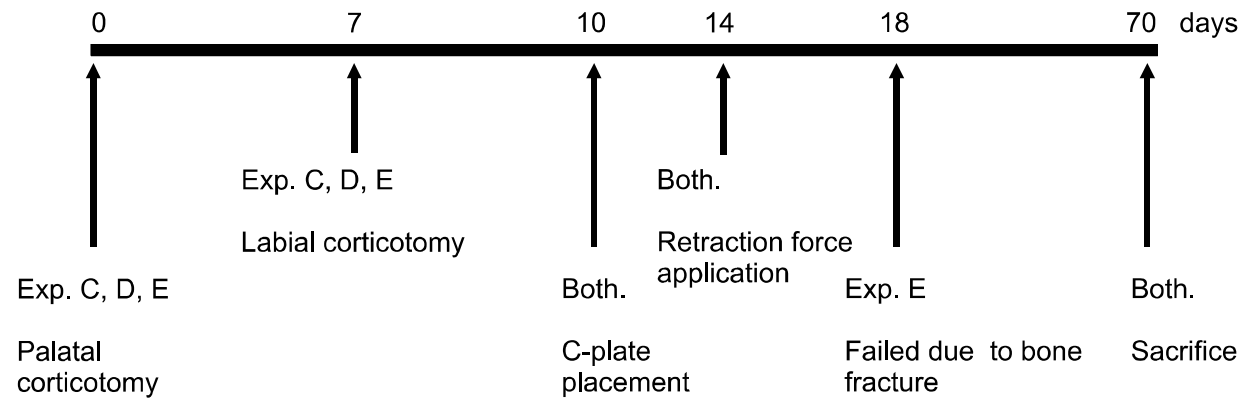

Fig 3. Schematic illustration of the experimental design. Exp., Experimental group; Both., control group (Group I) and experimental group (Group II).

Table 1. Velocity of en masse retraction

\begin{tabular}{|c|c|c|c|c|c|}
\hline & & \multicolumn{2}{|c|}{ Group I (control) } & \multicolumn{2}{|c|}{ Group II (experimental) } \\
\hline & & $\mathrm{A}$ & $\mathrm{B}$ & $\mathrm{C}$ & $\mathrm{D}$ \\
\hline \multirow[t]{3}{*}{ Rt } & Initial space $(\mathrm{mm})$ & 5.27 & 5.50 & 5.50 & 5.20 \\
\hline & Amount of retraction $(\mathrm{mm})$ & 2.20 & 2.00 & 3.50 & 4.00 \\
\hline & Velocity (mm/day) & 0.040 & 0.036 & 0.063 & 0.071 \\
\hline \multirow[t]{3}{*}{$\mathrm{Lt}$} & Initial space $(\mathrm{mm})$ & 5.27 & 5.60 & 5.30 & 5.30 \\
\hline & Amount of retraction $(\mathrm{mm})$ & 2.20 & 2.10 & 4.00 & 4.20 \\
\hline & Velocity (mm/day) & 0.040 & 0.038 & 0.071 & 0.075 \\
\hline
\end{tabular}

Rt, Right side; Lt, left side. 
for another $24 \mathrm{~h}$ at $37^{\circ} \mathrm{C}$. Subsequently, the teeth were mounted on an aluminum stub and coated with gold using a gold ion sputter for viewing under the scanning electron microscope (Hitachi S-2300, Hitachi, Ltd., Tokyo, Japan). Images were obtained at $\times 300$ and $\times 1,000$ magnifications.

Differences in the rates of en masse movements between the control and experimental groups were statistically compared using an independent Student's $t$ test. The right and left side tooth movement rates were considered as independent samples.

\section{RESULTS}

\section{Clinical and radiographic observations}

In animal A of Group I, the amount of space closure between the third incisor and canine was $2.20 \mathrm{~mm}$ on both sides after 56 days of active en masse retraction (Table 1). This value equates to a retraction rate of $0.040 \mathrm{~mm} /$ day. The average retraction rate of animal B was $0.037 \mathrm{~mm} /$ day (Table 2). In Group II, the retraction rate was $0.67 \mathrm{~mm} /$ day and $0.73 \mathrm{~mm} /$ day for animals $\mathrm{C}$ and $\mathrm{D}$ respectively. These values were almost double those of Group I, indicating that the en masse movement rate was faster in Group II. An independent $t$ test revealed statistically significant difference in the en masse movement rate between the control and experiment groups.

\section{Histological observation using hematoxylin and eosin staining}

As expected, numerous osteoclasts and a few osteoblasts were detected surrounding the alveolar bone

Table 2. Statistical comparison of en masse retraction velocity between control group and experimental group using independent t-test

\begin{tabular}{cccc}
\hline & $\begin{array}{c}\text { Control } \\
\text { group }\end{array}$ & $\begin{array}{c}\text { Experimental } \\
\text { group }\end{array}$ & $p^{\text {-value }}$ \\
\hline \hline Velocity & $0.0385 \pm$ & $0.07 \pm$ & $0.000^{*}$ \\
$(\mathrm{~mm} /$ day $)$ & 0.001914854 & 0.005033223 & \\
\hline
\end{tabular}

${ }^{*} p<0.05$. along the palatal side (pressure side) of the retracted maxillary incisor (Figs 4 and 5). In addition, a few osteoclasts were detected in the areas of discontinuous cementum and periodontal ligament (PDL), leading to a rough and irregular root surface on the pressure side. The surface irregularity on the root surface correlated with the pattern of resorbed cementum exposing the dentin to the PDL. In addition, the periodontal fibers were often detached from the surrounding alveolar bone. These findings were commonly noted on the pressure sides during teeth movement in Group I. On the other hand, in Group II, no osteoclasts were found on the cementum or dentin, although multiple osteoclasts were detected surrounding the alveolar bone facing the palatal side of the retracted incisor. In Group II, there was no evidence of root resorption on the compression side of the retracted incisor. In addition, the PDL space between the surrounding alveolar bone and the root surface was more intact and consistent in thickness in Group II compared to that in Group I (Fig $6 \mathrm{~A})$. The buccal side (tension side) of the retracted

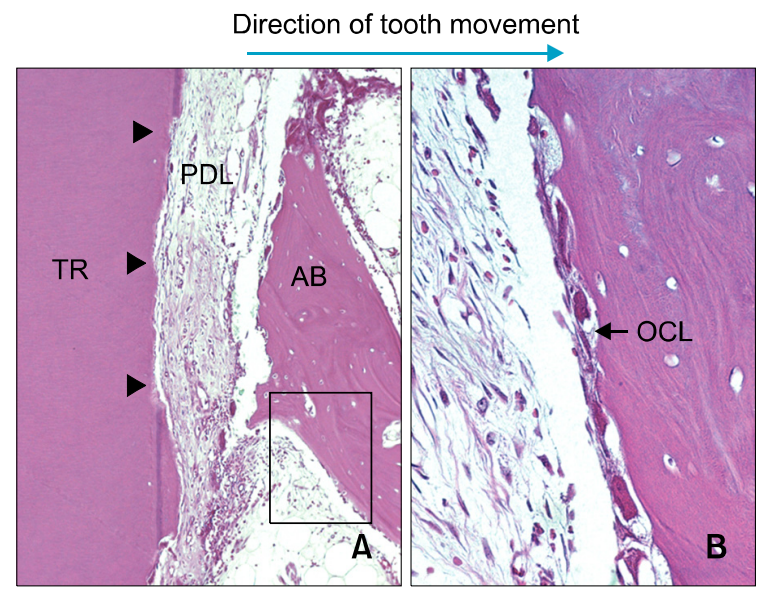

Fig 4. Microphotographs of periodontal tissue on a labio-lingual section of the retracted maxillary anterior tooth (hematoxylin and eosin (H\&E) stain) for lingual area (pressure side) for group I (dog A). A, Periodontal ligamental tissue was lost and root cementum was also partly absent. Note the demarcation line between root dentin and cementum was partially lost (H\&E, $\times 100)$. Black framed area is magnified in B. Short black arrow heads show root resorption; B, osteoclasts were observed on the surface of resorbing alveolar bone (H\&E, $\times$ 400). AB, Alveolar bone; PDL, periodontal ligament; $\mathrm{TR}$, tooth root; OCL, osteoclasts. 


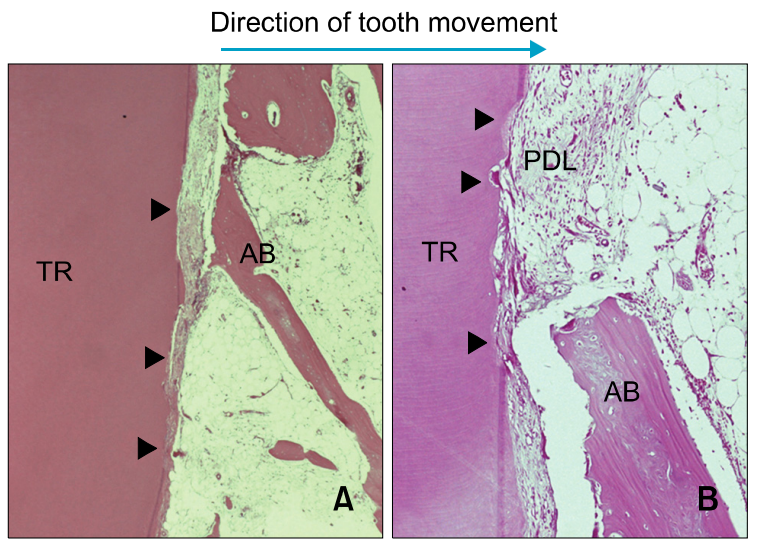

Fig 5. Microphotographs of periodontal tissue on the lingual area (pressure side) for group I (dog B). A, Odontoclasts were observed on the surface of the resorbing tooth surface. Attachment loss of periodontal ligament and loss of cementum continuity are seen (H\&E, $\times 40)$; B, higher magnification view of compression side $(H \& E, \times 100)$. Short black arrow heads show root resorption. AB, Alveolar bone; PDL, periodontal ligament; TR, tooth root.

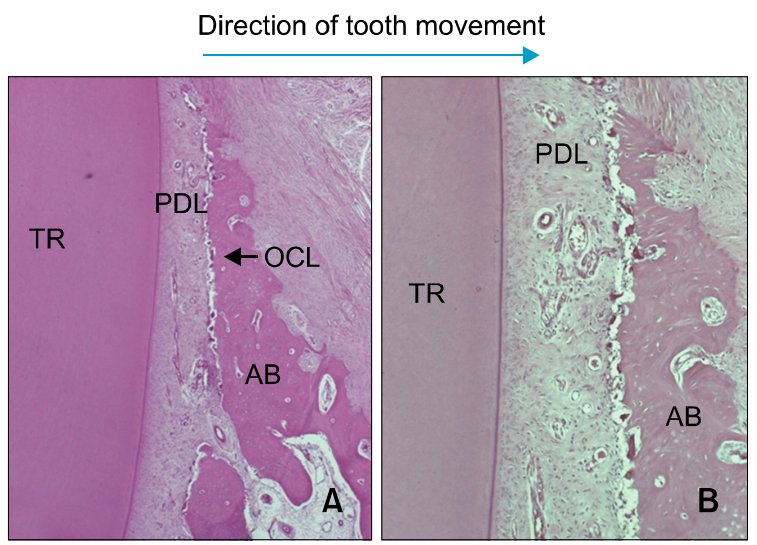

Fig 6. Microphotographs of periodontal tissue on the lingual area (pressure side) for group II (dog C). A, Many osteoclasts are observed on the surface of alveolar bone, but no root resorption is observed. Note the intact continuous cementum line of the dental root $(\mathrm{H} \& \mathrm{E}, \times 40)$; $\mathrm{B}$, higher magnification view of the compression side (H\&E, × 100). AB, Alveolar bone; PDL, periodontal ligament; TR, tooth root; OCL, osteoclasts.

maxillary incisor in Group I showed large amounts of newly formed osteoid-like tissue along with prominent vascularization in the direction of tooth movement (Fig 7). Group II showed similar histologic findings, but the extent and activity of new bone formation was rela-

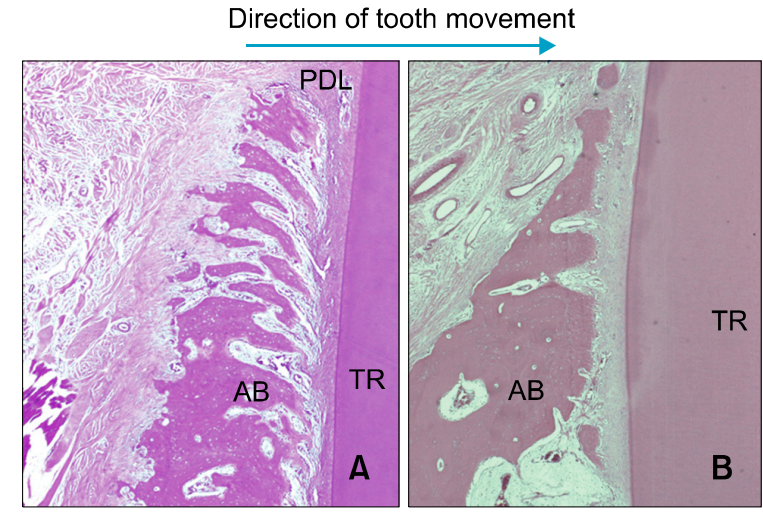

Fig 7. Microphotographs of periodontal tissue on the labial area (tension side) for group I (dog B) and group II (dog C). A, Many osteoblasts are observed on the surface of alveolar bone for group I (dog B). There are many capillaries in the marrow space of new bone formation, however, resorption activities of bone or root were not observed. Note that the newly formed bone trabeculae were directed horizontally coinciding with the direction of tooth movement (H\&E, $\times 40)$; B, histologic features of group II ( $\operatorname{dog} \mathrm{C})$ were similar to Group I but with less extent of new bone formation (H\&E, $\times$ 40). AB, Alveolar bone; PDL, periodontal ligament; TR, tooth root.

tively less (Fig 7B). In groups I and II, no resorption on the cementum or alveolar bone was observed along the tension side of the retracted incisor. In addition, the orientation of the osteoid-like tissue was more prominent in Group I than in Group II (Fig 6B).

\section{Morphological evaluation performed using a scanning electron microscope}

In Group I, multiple resorptive lacunae that were similar in size but variable in depth were often found clustered on the root surface of the compressed side of the retracted incisor (Fig 8A and B). Each cluster seemed well circumscribed, with numerous circular structures within the clusters, implying potential exposure of the dentinal tubules. The root surface of the retracted incisors of Group II was considerably smoother and had a relatively even texture on the compression side of the root. Resorptive lacunae were not noted in Group II, and the root surface was evenly covered by the PDL tissue (Fig 8C and D). The unresorbed root surface morphology in Group I was 

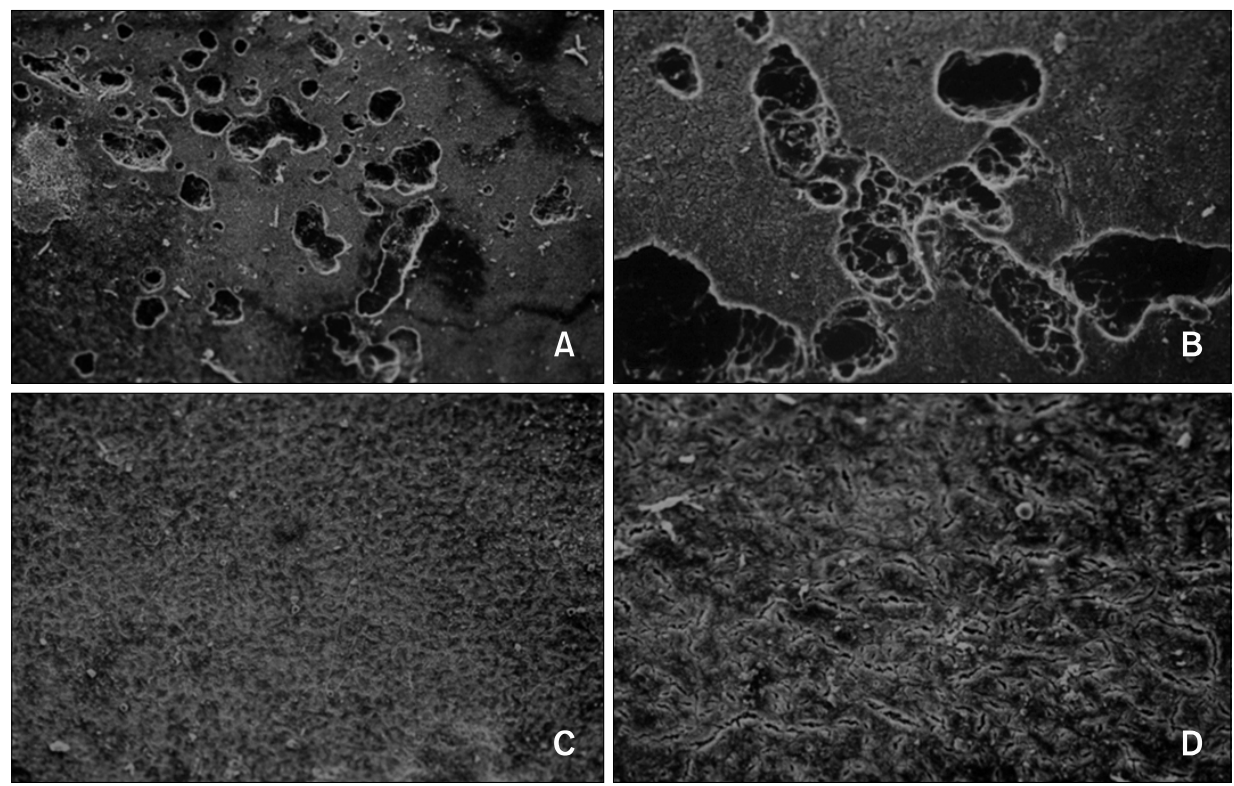

Fig 8. Scanning electron microscopy images of the dental root surface from group I (dogs A and B) and group II (dogs $C$ and D). A, Image of group I (dog A) shows resorption of cementum. Many resorption lacunae were clustered together and the sizes of lacunae were similar although the depths varied (SEM, $\times 100)$; $\mathbf{B}$, higher magnification image of group I $(\operatorname{dog} A)$ shows round resorption lacunae with definite boundaries. Several small holes were visible in the bottom of some lacunae which are considered to be dentinal tubules (SEM, $\times 300$ ); C, image of group II (dog D) shows smooth root surfaces without resorption lacunae (SEM, $\times 100)$; D, higher magnification of image of group II $(\operatorname{dog} D)$ at SEM, $\times 300$.

smooth (Fig 8A and B), whereas that in Group II showed a network of fine grooves. Since the root samples for SEM were processed in a similar manner for both groups, procedural variation could be ruled out as a source of error. Resorption of hard tissue is preceded by elimination of adjacent soft tissues by enzymatic digestion with enzymes such as matrix metalloproteinases. In Group I, root resorption provided a smoother appearance to the root surface due to the enzymatic elimination of adjacent collagen fibers. Since root resorption did not occur in Group II, the collagen fibers covering the root surface remained intact. The difference in the root surface morphology between a resorbed and unresorbed root can be understood by subjecting an unresorbed root sample to collagenase treatment.

\section{DISCUSSION}

Orthodontic treatment combined with corticotomy has been shown to shorten the overall orthodontic treatment time for complex cases that were previously treated only by orthognathic surgery. One theory is that corticotomy triggers a cascade of physiological anabolic events, leading to an accelerated bone turnover rate and decreased regional bone density. This effect was termed by Frost as regional acceleratory phenomenon (RAP). ${ }^{15,16}$ RAP is localized to the surgical site and its immediate surrounding tissue instead of the entire body. Considering the data obtained from animal studies, it should be able to observe RAP in humans within a few days of surgical intervention, peak around 1 to 2 months, and may require 6 to more than 24 months for disappearence. ${ }^{15}$ Therefore, adjusting orthodontic appliances more actively and frequently is recommended within the first 6 months following surgical intervention to facilitate the RAP effect and reduce the orthodontic therapy time. Another theory is that corticotomy physically decreases the mechanical resistance of tooth movement through the bone by effectively reducing cortical bone density of the side toward which the tooth is moved. ${ }^{7,8,17,18}$ The third theory is that a 
complete linear-shaped decortication around a target segment can provide a focal-stress-bearing region when a heavy orthopedic force is applied. This in turn results in a medullary bone-bending effect at the decortication area rather than in the periodontal ligament space. This effect becomes apparent as the repositioning of the dental segment occurs in a short period of time..$^{9-12,19,20}$

SSO is a novel orthodontic treatment method that is primarily based on the third hypothesis in combination with the other 2 concepts. SSO has been reported by many clinical case studies to be an effective and efficient treatment modality for correcting adult bimaxillary protrusion without requiring orthognathic surgery or anterior segmental osteotomy. ${ }^{12,14,19}$ However, the potential negative effects of SSO on the periodontium (tooth, PDL, alveolar bone, and gingiva) have not been fully understood. The present study provides sufficient evidence in this regard because it is the first animal study that shows the effects of SSO on the periodontium, and the results seem promising.

The depth of corticotomy was determined to be adequate when only minor bleeding occurred upon entry into the trabecular bone. However, beagle dogs have very thick cortical alveolar bones and relatively thin medullary alveolar bones. Hence, the corticotomy procedure was quite difficult to perform even by an experienced oral and maxillofacial surgeon. The maxillary anterior segment fracture in animal $\mathrm{E}$ during en masse retraction, therefore, might be attributed to a more aggressive corticotomy rather than to an excessive retraction force.

To maximize the effects of corticotomy for orthodontic tooth movement, active orthodontic en masse retraction of the 6 maxillary incisors was initiated 1 week after the second corticotomy procedure by using a bonded palatal retractor. The retractive force used in the current study was $250 \mathrm{~g}$ per side, and a direction was chosen such that the force was applied through the center of the retracted segment to induce more translational segmental movement. The force level was decided on the basis of that used in a previous animal study by Yoshikawa, in which a headcap was used after maxillary block corticotomy in monkeys, followed by application of $400 \mathrm{~g}$ of retractive force on either side. $^{21}$
Since there are obvious differences in the maxillofacial anatomy of beagles and humans, direct extrapolation of the results of this study would be difficult. Nonetheless, several very interesting findings were noted in this study. The overall rate of en masse retraction of the 6 maxillary incisors in Group II was $0.070 \mathrm{~mm} /$ day, whereas that of Group I was 0.0385 $\mathrm{mm}$ /day. This means that the rate was approximately 1.8 times faster in Group II. This finding corroborates the clinical efficiency of SSO reported in previous literature. Another interesting point was that the retraction in Group II primarily resulted from the bonebending movement along the perisegmental corticotomy line, ${ }^{7}$ whereas that in Group I resulted from the pure dental movement within the surrounding alveolar bone (Unpublished data: The bone-bending effect measured by cephalometric analysis was found to be consistent in 24 patients who underwent maxillary en masse retraction with the SSO protocol). ${ }^{22}$ In addition to our unpublished data, the preliminary histological data obtained in this study further supports this fact. In Group I, in which the teeth were retracted using a heavy orthopedic force without perisegmental corticotomy, multiple osteoclasts were present not only in the pressured alveolar bone area but also directly on the root surface. This resulted in severe irregularity of the root surface, indicating the occurrence of root resorption in Group I (Figs 4 and 5). On the other hand, the pressure-sided root surface in Group II did not show any osteoclasts on the root surface (Fig 6). In other words, the cementum and dentin of the teeth in Group II showed no root resorption because of the heavy orthopedic force. This was further confirmed by SEM evaluation of the tooth surface, showing multiple clustered resorptive lacunae in Group I but none in Group II (Fig 8). Our previous understanding of the biological mechanisms underlying orthodontically induced root resorption does not completely explain this finding. Nevertheless, during corticotomy, some part of the periodontal ligament would sustain forces sufficient enough to occlude blood vessels, resulting in hyalinization changes that are known to induce root resorption. Microscopic observation showed hyalinization-like zones in the samples subjected to corticotomy but no histologic features of root resorption. Iino et al. ${ }^{23}$ performed 
corticotomy on the third premolars of beagle dogs by applying orthodontic force. They reported hyalinization of the periodontal ligament without histologic signs of root resorption. They suggested that root resorption might not have occurred due to the disappearance of the lag phase. That is, less hyalinization with relatively faster elimination resulted in no root resorption. Although their explanation seems reasonable, further evidence is required to understand the biologic mechanism underlying root resorption. Due to the small sample size and preliminary nature of this study, it would be difficult to conclude that corticotomy-assisted orthodontics does not induce root resorption. However, the corticotomy procedure can be considered beneficial for orthodontic patients who have roots that are prone to resorption or have short roots.

The histologic analysis showed prominent linear osteoid development on the tension-sided alveolar bone area in Group I but not as much in Group II. This indicates very active dentoalveolar response by the teeth movement in Group I (Fig 7). More importantly, the periapical area of the retracted incisors, where the perisegmental corticotomy was made, showed numerous plasma-rich osteoblasts, suggesting active bone remodeling at the corticotomy site. Therefore, unlike the conventional retraction method, speedy retraction following SSO protocol might allow early movement of the retracted dental segment, without causing much damage to the teeth and the periodontium. However, further investigation involving more subjects is warranted to statistically confirm these data. No major abnormalities such as gingival recession or alveolar bone loss were noted in the visual and radiographic evaluations in both the groups. In addition, no pathological changes were noted in the dentin and pulp in Group II. This was in concordance with the findings of previous studies reporting uncompromised blood supply to the teeth and rapid tooth movement following corticotomy. ${ }^{23,24}$

As clinicians, we frequently face many challenges in finding out ways (1) to minimize damage to the periodontal tissue, (2) to reduce treatment time, (3) to design more esthetic orthodontic appliances, and (4) to avoid orthognathic surgery for treating very complex skeletally induced malocclusions in adults. Our current preliminary data suggest that SSO may be considered as one of the most effective orthodontic solutions to these problems because of the following reasons. First, SSO causes less damage to the periodontium during the retraction of the maxillary anterior teeth because the retraction primarily results from the bone-bending movement along the perisegmental corticotomy line and not from the tooth movement per se. This minimizes the risk of severe root resorption during the en masse retraction. Second, the retraction process using the SSO protocol occurs almost twice as faster as the conventional orthodontic retraction. Therefore, it contributes to reducing the overall orthodontic treatment time. Third, the appliances used in the SSO protocol (C-lingual retractor and C-palatal mini-plate) overcome the need for orthodontic therapy during en masse retraction, reducing the time required for labial fixation of orthodontic appliances. ${ }^{25}$ Fourth, the amount of retraction of a dental segment facilitated by SSO is much greater than that possible by most conventional orthodontic retraction methods. Hence, SSO overcomes the need of orthognathic surgery or anterior segmental osteotomy under general anesthesia for the correction of skeletal maxillary protrusion in adults.

In this study, we showed that corticotomy is an efficient method for en masse retraction and for avoiding marked root resorption. In future, studies with larger sample size and more comprehensive analysis methods are warranted to confirm the efficiency and safety of corticotomy procedures. The effects of corticotomy on the tooth pulp vitality and tooth moving rate when varying amounts of force are applied also need to be investigated.

\section{CONCLUSION}

The results of this preliminary animal study provide evidence that corticotomy-assisted en masse retraction of the maxillary anterior teeth allows early teeth movement without causing noticeable periodontal damage and root resorption.

\section{REFERENCES}

1. Melsen B. Limitations in adult orthodontics. In: Melsen B 
editor. Current controversies in orthodontics. 1st ed. Hanover Park, IL: Quintessence Publishing Co, Inc.; 1991. p. 147-80.

2. Vardimon AD, Oren E, Ben-Bassat Y. Cortical bone remodeling/tooth movement ratio during maxillary incisor retraction with tip versus torque movements. Am J Orthod Dentofacial Orthop 1998;114:520-9.

3. Bojrab DG, Dumas JE, Lahrman DE. JCO/interviews Dr. David G. Bojrab, Dr. James E. Dumas, Dr. Don E. Lahrman on surgical-orthodontics. J Clin Orthod 1977;11:330-42.

4. Wilcko WM, Wilcko T, Bouquot JE, Ferguson DJ. Rapid orthodontics with alveolar reshaping: two case reports of decrowding. Int J Periodontics Restorative Dent 2001;21:9-19.

5. Chen YR, Yeow VK. Multiple-segment osteotomy in maxillofacial surgery. Plast Reconstr Surg 1999;104:381-8.

6. Kole $\mathrm{H}$. Surgical operations on the alveolar ridge to correct occlusal abnormalities. Oral Surg Oral Med Oral Pathol 1959; 12:413-20.

7. Wilcko MT, Wilcko WM, Pulver JJ, Bissada NF, Bouquot JE. Accelerated osteogenic orthodontics technique: a 1-stage surgically facilitated rapid orthodontic technique with alveolar augmentation. J Oral Maxillofac Surg 2009;67:2149-59.

8. Wilcko MT, Wilcko WM, Murphy KG, Carroll WJ, Ferguson DJ, Miley DD, et al. Full-thickness flap/subepithelial connective tissue grafting with intramarrow penetrations: three case reports of lingual root coverage. Int $\mathrm{J}$ Periodontics Restorative Dent 2005;25:561-9.

9. Suya H. Corticotomy in orthodontics. In: Hosl E, Baldauf A editors. Mechanical and biological basics in orthodontic therapy. Heidelberg, Germany: Huthig Buch; 1991, p. 207-26.

10. Lee BS, Hwang HW, Chung KR. Clinical use of corticotomies in adult orthodontics. J Korean Assoc Maxillofac Plast Reconstr Surg 1999;21:303-11.

11. Chung KR, Oh MY, Ko SJ. Corticotomy-assisted orthodontics. J Clin Orthod 2001;35:331-9.

12. Chung KR, Mitsugi M, Lee BS, Kanno T, Lee W, Kim SH. Speedy surgical orthodontic treatment with skeletal anchorage in adults--sagittal correction and open bite correction. J Oral Maxillofac Surg 2009;67:2130-48.

13. Kim S, Park Y, Chung K. Severe anterior open bite malocclusion with multiple odontoma treated by $\mathrm{C}$-lingual retractor and horseshoe mechanics. Angle Orthod 2003;73:206-12.

14. Kim SH, Lee KB, Chung KR, Nelson G, Kim TW. Severe bi- maxillary protrusion with adult periodontitis treated by corticotomy and compression osteogenesis. Korean J Orthod 2009;39: 54-65.

15. Frost HM. The biology of fracture healing. An overview for clinicians. Part I. Clin Orthop Relat Res 1989;248:283-93.

16. Frost HM. The biology of fracture healing. An overview for clinicians. Part II. Clin Orthop Relat Res 1989;248:294-309.

17. Gwack C, Kim SS, Park SB, Son WS, Kim YD, Jun ES, et al. The expression of MMP-1, -8 , and -13 mRNA in the periodontal ligament of rats during tooth movement with cortical punching. Korean J Orthod 2008;38:187-201.

18. Park WK, Kim SS, Park SB, Son WS, Kim YD, Jun ES, et al. The effect of cortical punching on the expression of OPG, RANK, and RANKL in the periodontal tissue during tooth movement in rats. Korean J Orthod 2008;38:159-74.

19. Chung KR, Kim SH, Lee BS. Speedy surgical-orthodontic treatment with temporary anchorage devices as an alternative to orthognathic surgery. Am J Orthod Dentofacial Orthop 2009;135:787-98.

20. Kim DH, Park YG, Kang SG. The effects of electrical current from a micro-electrical device on tooth movement. Korean J Orthod 2008;38:337-46.

21. Yoshikawa Y, Deguchi T, Eda S. Pulpal and radicular changes following maxillary subapical corticotomy. Endod Dent Traumatol 1992;8:245-7.

22. Lee JK, Chung KR, Baek SH. Treatment outcomes of orthodontic treatment, corticotomy-assisted orthodontic treatment, and anterior segmental osteotomy for bimaxillary dentoalveolar protrusion. Plast Reconstr Surg 2007;120:1027-36.

23. Iino $S$, Sakoda $S$, Ito $G$, Nishimori $T$, Ikeda $T$, Miyawaki $S$. Acceleration of orthodontic tooth movement by alveolar corticotomy in the dog. Am J Orthod Dentofacial Orthop 2007;131: 448.e1-8.

24. Wang L, Lee W, Lei DL, Liu YP, Yamashita DD, Yen SL. Tisssue responses in corticotomy- and osteotomy-assisted tooth movements in rats: histology and immunostaining. Am J Orthod Dentofacial Orthop 2009;136:770.e1-11.

25. Chung KR, Kook YA, Kim SH, Mo SS, Jung JA. Class II malocclusion treated by combining a lingual retractor and a palatal plate. Am J Orthod Dentofacial Orthop 2008;133: $112-23$. 